\title{
High Level Aminoglycoside Resistance (HLAR) in Enterococcus Species Isolated from Various Clinical Specimens
}

\author{
Sheetal Sharma ${ }^{1}$, Prasanna Gupta ${ }^{2}$ and Suman Rishi ${ }^{2}$ \\ ${ }^{1}$ Medical Microbiology, ${ }^{2}$ Department of Microbiology, National Institute of Medical Sciences \\ \& Research Jaipur, NIMS University (Rajasthan) India- 303121 \\ *Corresponding author
}

\section{Keywords}

High level aminoglycoside resistance,

Enterococcus Infections, E-test

\section{Article Info}

Accepted:

24 November 2018

Available Online:

10 December 2018

\section{A B S T R A C T}

Enterococci are the most aerobic and facultative anaerobe, gram positive cocci. They constitute a part of normal intestinal flora. However they also occupy other sites such as oral cavity, skin etc. Enterococci, which earlier considered as low grade pathogens, has now emerged as one of the most important nosocomial pathogens worldwide and are associated with high mortality. Study was carried out in the Department of Microbiology, National Institute of Medical Sciences \& Research Jaipur (India) between December 2016 and December 2017. The isolated Enetrococci are then tested for routine antibiotics sensitivity by disc diffusion method including High level Gentamicin and Streptomycin disc. Further confirmation of High level Gentamicin and Streptomycin resistance by E-test (MIC). We consider MIC test as gold slandered method. A total 110 Enterococcus isolates are obtained from various clinical specimens such as Blood, Urine, Pus. Among 110 Enterococcus species, 78 species are Enterococcus faecalis and 32 species are Enterococcus faecium. Out of 110 Enterococcus isolates 36 (32.7\%) are resistant to High level Gentamicin and $24(21.8 \%)$ are resistant to High level streptomycin by disc diffusion method and by E-test High level Gentamicin was 41 (37.2\%) and High level streptomycin was $27(24.5 \%)$ found. Prevalence of HLAR is high and could be a serious problem in hospital setup, screening for high level aminoglycoside resistance must be included in routine antibiotic susceptibility reporting for Enterococcal isolates.

\section{Introduction}

Enterococci are gram positive cocci, known only as intestinal commensals with little significance have evolved as deadly pathogens over last two decades. Enterococcal infections is always difficult to treat due to inherent resistance to many commonly used antibiotics like aminoglycosides, cephalosporins etc (Marothi et al., 2014). Enterococci, initially considered as normal commensals of intestinal tract, have recently emerged as a medically important pathogen. Incidence of eneterococcal infections is significantly high in patient suffering from urinary tract infection, blood stream infection, and surgical site infections. Nosocomial eneterococcal infection is also common in organ transplant recipient's cancer patients and debilitated patients receiving broad spectrum antibiotics 
(Sadar et al., 1994). Enterococci have traditionally been treated with cell wall active agents in combination with an aminoglycoside. However resistance to low and high level aminoglycosides has been reported. Resistance to beta lactam antibiotics and vancomycin by some strains together with High level aminoglycoside resistance (Patterson et al., 1998).

The present study was aimed to detecting the antimicrobial resistance pattern among enterococcus isolates obtained from clinical specimen in a tertiary care hospital in Jaipur with special emphasis on high level aminoglycosides and prevalence of enterococcus spp. in our hospital.

\section{Materials and Methods}

The study was carried out of one year December 2016 to December 2017 at the Department of microbiology, National Institute of Medical Sciences \& Research, NIMS University Jaipur (Rajasthan). Various clinical specimens Urine, Pus, Blood, Body fluids were taken from patients attending NIMS hospital. Specimen were collected in a sterile, proper labelled container with aseptic precautions and processed as per standard microbiological procedures.

\section{Organism identification by Henry et al.,} (1998)

All samples were screened for the pus cells and organism. Specimen was culture on Blood agar and MacConkey and incubated for $37^{\circ} \mathrm{C}$ for 24 hours. Growth was then processed for gram staining and catalase test. Gram positive cocci arranged in pairs showing catalase negative were considered as streptococcus species. Speciation of Enterococcus species done by grams staining, colony morphology, culture characteristics of the colonies and biochemical tests (Bile esculin hydrolysis test,
Pyrrolidonyl Arylamidase, resistance to bacitracin and optochin, growth at $6.5 \% \mathrm{NaCl}$, growth at $37^{\circ} \mathrm{C}$ and $45^{\circ} \mathrm{C}$, Hippurate hydrolysis test, sugar fermentation test). Grams stain smear shows gram positive cocci $1-1.5 \times 0.5 \mu \mathrm{m}$, oval shaped arranged in pairs and short chains.

\section{Antibiotic susceptibility testing}

Antibiotic susceptibility testing was carried on Brain Heart Infusion (BHI) agar by modified Kirby-Bauer disc diffusion method (CLSI $28^{\text {th }}$ ed 2018). High level aminoglycoside resistance (HLAR) method was detected by following methods.

\section{Disc diffusion method}

Colonies of Enetrococcus was inoculated into broth and incubated at $37^{\circ} \mathrm{C}$ for 4 hours. Growth was indicated by the appearance of turbidity in the medium. Turbidity of the medium was compared with 0.5 McFarland tube. Lawn culture was performed on BHI agar plate with the help of sterilized swab, Gentamicin and Streptomycin drugs was inoculated with a sterile - forceps and then incubated.

\section{Minimum Inhibitory Concentration (MIC) method}

Minimum Inhibitory Concentration of Gentamicin and Streptomycin was determined by E-test. The strains which were resistant by disc diffusion method were checked by MIC. The colonies were inoculated in BHI broth. Growth was indicated by the appearance of turbidity which was compared with 0.5 McFarland tube. Lawn culture was performed on BHI agar plate with sterile swab and Estrip was inoculated on BHI plate and incubated. All the result was interpreted according to CLSI guidelines 2018. 


\section{Results and Discussion}

In our study a total of 110 Enetrococcus were isolated in a period of one year 2016 to December 2017. Among 110 Enterococcus species, 78 species are Enterococcus faecalis and 32 species are Enterococcus faecium.

Highest prevalence of Enterococcus was seen in females $74(67.2 \%)$ followed by males 36 $(32.7 \%)$. The maximum percentage of isolation was seen among the age group 40-60 years.

In our study maximum Enterococcus isolate from urine specimen 69 (62.7\%), followed by Pus 21 (19\%), Blood 12 (10.9\%), Others 08 $(7.2 \%)$.
Maximum isolation of Enterococcus isolates was isolated from urine specimen. It indicates that urinary tract infections are the most common infections caused by Enterococci in this set up (Table 1-2).

All the enterococcal isolates were subjected to test for High level Aminoglycoside resistance by two methods name Gentamicin and Streptomycin high concentration disc diffusion method and MIC method. We Consider E test is gold slandered method. The results of Gentamicin and Streptomycin by high concentration discs $120 \mu \mathrm{g}$ and $300 \mu \mathrm{g}$ has shown in table 3 and 4.

Table.1 Showing sample distribution

\begin{tabular}{|l|c|c|}
\hline \multicolumn{1}{|c|}{ Specimen } & No. Of Isolates & Percentage (\%) \\
\hline Urine & 69 & $62.7 \%$ \\
\hline Pus & 21 & $19 \%$ \\
\hline Blood & 12 & $10.9 \%$ \\
\hline Others (Body fulids, sputum) & 08 & $7.2 \%$ \\
\hline Total & 110 & $100 \%$ \\
\hline
\end{tabular}

Table.2 Showing Enterococcus species distribution

\begin{tabular}{|c|c|c|}
\hline Entero.spp & No. Of Isolates & Percentage (\%) \\
\hline Enterococcus faecalis & 78 & $70.9 \%$ \\
\hline Enterococcus faecium & 32 & $29.0 \%$ \\
\hline Total & 110 & $100 \%$ \\
\hline
\end{tabular}

Table.3 Showing high level aminoglycoside resistance in Enterococcus species by disc diffusion method

\begin{tabular}{|c|c|c|}
\hline Antibiotics & Resistance (n-110) & Percentage \\
\hline $\begin{array}{l}\text { High level Gentamicin } \\
(120 \mu \mathrm{g})\end{array}$ & 34 & $30.9 \%$ \\
\hline $\begin{array}{l}\text { High level Streptomycin } \\
(300 \mu g)\end{array}$ & 17 & $15.4 \%$ \\
\hline
\end{tabular}


Table.4 Showing high level aminoglycoside resistance in Enterococcus species by MIC method

\begin{tabular}{|c|c|c|}
\hline Antibiotics & Resistance (n-110) & Percentage \\
\hline High level Gentamicin (0.064-1024 $\mathrm{mcg} / \mathrm{ml})$ & 27 & $24.5 \%$ \\
\hline High level Streptomycin (0.016-256 $\mathrm{mcg} / \mathrm{ml})$ & 12 & 10.90 \\
\hline
\end{tabular}

Table.5 Showing antibiotic sensitivity of Enterococcus species to other antibiotics

\begin{tabular}{|c|c|c|}
\hline Antibiotic Used & Sensitivity & Resistant \\
\hline Ampicillin (10ug) & $39(53.4 \%)$ & $71(64.5 \%)$ \\
\hline 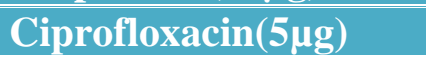 & $62(56.3 \%)$ & $48(43.6 \%)$ \\
\hline Norfloxacin* & $18(26.0 \%)$ & $51(73.9 \%)$ \\
\hline Nitrofurantoin* $(30 \mu \mathrm{g})$ & $28(40.5 \%)$ & $41(59.4 \%)$ \\
\hline 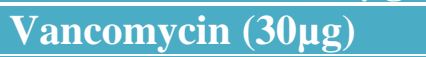 & $110(100 \%)$ & 00 \\
\hline Linezolid (30ug) & $110(100 \%)$ & 00 \\
\hline
\end{tabular}

*Used for urine isolates only (69 samples)

In the present study maximum number of Enterococci were isolated from urine $(62.7 \%)$ followed by Pus (19\%). This is slightly lower than Ruoff et al., (1990) who isolate maximum number of Enterococci from urine $(68.2 \%)$. In another study of Talebi et al., (2007) also reported maximum number of Enterococci from urine (85\%) followed by Pus (15.5\%). Karmarkar et al., (2004) isolated $47.14 \%$ Enterococci from urine sample and described that urinary tract as commonest site of isolation of Enterococci. The maximal enterococcal urine isolation could be due to structural abnormalities in the urinary tract, indwelling catheter of following any instrumentation.

Antibiotic resistance among Enterococci is global problem. Antibiotic resistance in Enterococci is intrinsic or acquired. In our study the highest resistant is seen against Ampicillin 64.5\%, another study of Salem Bekhit et al., (2012) also report high resistance of ampicillin $70.4 \%$ in E. faecium. In our study ciprofloxacin resistant is $43.6 \%$, another study of Sarika Jain et al., (2011) also reported high resistant of ciprofloxacin in enterococci $75 \%$.
According to our study the highest sensitivity pattern is shown in vancomycin and linezolid for all samples and for urinary isolates nitrofurantoin shows $40.5 \%$ sensitive and norfloxacin shows $26 \%$ sensitive.

In our study High Level Gentamicin resistance by $\mathrm{E}$ test is $25.4 \%$ and high level Streptomicin resistance is $15.4 \%$. In another study of Sanal C. Fernandes et al., (2013) report $53 \%$ resistance of High Level Gentamicin and 34.1 resistance in high level Streptomicin resistance. The presence of HLGR is predictive of the loss of the synergy between Gentamicin and a cell wall active agent such as ampicillin or vancomycin Murray et al., (1998) (Table 5).

In conclusion Enetrococcus infections are mainly associated with nosocomial infection and increase incidence mainly due to indiscriminate use of broad spectrum of antibiotics. Proper infection control practices need to prevent nosocomial origin of Enterococci. This study emphasizes the need to screen for HLAR in patients suffering from eneterococcal infections as a routine screening for to detect HLGR and HLSR as 
this will help to limit the spread of resistance and have a surveillance pattern.

\section{References}

CLSI: Clinical and Laboratory Standard Institute. Performance Standards for Antimicrobial Susceptibility Testing. 28 ed. Wayne. PA: Clinical and Laboratory Standard Institute; 2018.

Henry, D. 1998. Isenberg. Essential Procedures for Clinical Microbiology. Collection, Transport and Manipulation of Clinical Specimens. ASM Press Washington, D.C. Pp. 3-35.

Karmarkar M.G. Enterococcal infections with special reference to phenotypic characterization \& drug resistance. Indian J Med Res Suppl May 2004; 119: 22-25.

Marothi YA, Agnihotri H, Dubay D. Enterococcal resistance - An overview. Indian Journal of Medical Microbiology. 2005; 23(4): 214-19.

Murray BE. Diversity among multidrugresistant enterococci. Emerg Infect Dis 1998; 4: 37-47.

Patterson, J.E., Zervos, M. High level gentamicin resistance in Enterococcus: microbiology, genetic basis and epidemiology. Rev. Infect. Dis., 12:6
44-51.

Ruoff LK, Spargo DJ, Ferraro JM "Species identification of Enterococci Isolates from Clinical Specimens" J Clin Microbiol 1990; 28: 435-437.

Sadar, H.S., Pfaller, M.A., Tenover, F.C., Hollis, R.J., Jones, R.N. 1994. Evaluation and characterization of multiresistant Enterococcus faecium from 12 US medical centers. J. Clin. Microbiol., 32: 2840 -2.

Salem-Bekhit MM et al., Prevalence and antimicrobial resistance pattern of multidrug-resistant enterococci isolated from clinical specimens. Indian Journal of Medical Microbiology. 2012; 30(1): 44-5.

Sanal C. Fernandes et al, "Drug resistance \& virulence determinants in clinical isolates of Enterococcus species, IJMR, May 2013; 137: 981-985.

Sarika Jain et al, Clinico epidimiological profile and high level aminoglycoside resistance in enterococcal septicaemia from a tertiary care hospital in east Delhi. International J of Basic Medical Research. Jul-Dec 2011; 1(2): 354-360.

Talebi M. Characterization of Vancomycin Resistant Enterococcus faecium" Iranian JPubl Health. 2007; 36(4): 2025.

\section{How to cite this article:}

Sheetal Sharma, Prasanna Gupta and Suman Rishi. 2018. High Level Aminoglycoside Resistance (HLAR) in Enterococcus Species Isolated from Various Clinical Specimens. Int.J.Curr.Microbiol.App.Sci. 7(12): 3178-3182. doi: https://doi.org/10.20546/ijcmas.2018.712.366 